\title{
Efectos secundarios gastrointestinales por antiinflamatorios no esteroideos y costes en el Sistema Nacional de Salud
}

Lanas A. Efectos secundarios gastrointestinales por antiinflamatorios no esteroideos y costes en el Sistema Nacional de Salud. An Med Interna (Madrid) 2001; 18: 561-563.

Los efectos secundarios originados por fármacos son una causa frecuente de morbilidad y de mortalidad. Estudios recientes señalan que tanto en Estados Unidos como en el Reino Unido la mortalidad asociada a efectos secundarios por fármacos ocupan un lugar destacado entre las 10 causas más frecuentes de muerte en esos países $(1,2)$, compitiendo con otras ampliamente conocidas como el cáncer, los accidentes de circulación, la patología cardiovascular, etc. La situación en nuestro país no debiera ser diferente, pues con el sistema que disponemos, el acceso a las medicinas esta garantizado para la casi totalidad de la población. A esta situación se suma en nuestro país la facilidad de acceso a los fármacos sin la correspondiente prescripción médica.

Uno de los grupos farmacológicos más vendidos y utilizados por la población en el mundo occidental es el de los analgésicos y los anti-inflamatorios no esteroideos (AINEs). Este hecho no es extraño, pues la patología osteoarticular se encuentra en la cima de las patologías que más afecta la calidad de vida de las personas (3) y los AINES son fármacos efectivos en la reducción del dolor y la inflamación en estos pacientes. Los efectos secundarios originados por los AINEs afectan a diversos órganos, pero los originados a nivel gastrointestinal son los de máxima frecuencia. Aproximadamente un 2-3\% de los pacientes que toman AINEs durante un año desarrollan una complicación gastrointestinal como hemorragia o perforación alta o baja. Durante ese periodo de tiempo un 5-10\% desarrollan úlceras sintomáticas y un 30-50\% desarrollan dispepsia que requiere atención médica (4).

Los gastos originados por estos efectos secundarios han sido cuantificados en diversos estudios y en diferentes países. Dados los diferentes sistemas de atención médica y los diferentes niveles de vida existentes aun dentro del mundo occidental, las estimaciones efectuadas en unos países no son enteramente extrapolables a otros, aunque las mayores diferencias globales se dan entre el sistema norteamericano y el europeo. En este número de Anales de Medicina Interna, Vargas y cols. presentan datos referentes al coste estimado en gastos directos que las complicaciones asociadas a AINEs en 1998 supusieron para el Sistema Nacional de Salud y que ascendían a 8.112 millones de pesetas (5). A pesar de que los autores han asumido que el porcentaje de pacientes que tomaron AINES, y que recogieron de forma retrospectiva, es igual al porcentaje de complicaciones atribuibles a AINEs, sin contar que a esa cifra hay que restar el porcentaje de consumo de AINEs en la población control, los costes estimados son muy similares a otro estudio publicado que hacía también referencia al año 1998 (6). A pesar de la diferente concepción entre lo que es complicación asociada a AINEs y complicación atribuible a AINEs, la coincidencia en cifras en ambos estudios se debe a que en el trabajo anteriormente referido se partía de cifras de asociación de complicaciones al consumo de AINE superiores (6). Estas cifras se basaban en datos de estudios prospectivos recogidos en el mismo área en donde se efectuaron los cálculos de los costes para todo el Sistema Nacional de Salud. De cualquier forma, la coincidencia en los datos no hacen sino reforzar el hecho que nuestro Sistema Nacional de Salud español se gasto entre 6.500 y 9.000 millones de pesetas en tratar las complicaciones originadas por AINEs en 1998. Las cifras se encuentran también dentro de las estimaciones efectuadas en otros países europeos (7).

A pesar de que estos costes en complicaciones suponen un gasto para nada desdeñable, los gastos más importantes asociados a la utilización de AINEs se derivan de las medidas que los facultativos tomamos para prevenir estos efectos secundarios y para tratar aspectos de menor relevancia como la dispepsia que puede estar presente hasta en el 50\% de los pacientes que toman AINEs. En este sentido en España durante el periodo referido de 1998 se gastó al menos 3,39 veces más en prevenir esas complicaciones y 1,3 veces más en tratar la dispepsia originada por los AINEs que en tratar las complicaciones. Iguales conclusiones pueden sacarse de datos publicados en Suecia donde se gastó 2,77 veces más en tratar la dispepsia que en tratar complicaciones, o en el Reino Unido donde se gastó casi 7 veces más en prevenir que en tratar las complicaciones (7). Los datos referidos a España en 1998 eran costes mínimos pues se estimó tasas de co-prescripción del $25 \%$. Con los datos actuales en los que se estima un $50 \%$ en coprescripción de AINE y gastroprotector, los datos se disparan y se estima un coste 10 veces más en prevenir una hemorragia que en tratarla (8). 
El aumento en el gasto no es intrínsicamente malo sino bueno, si este se asocia a un beneficio claro en la disminución de sufrimiento y en mejora de la calidad de vida de los pacientes. Si este gasto se asocia además a una reducción en mortalidad, el beneficio será completo. No debe olvidarse que se ha estimado que el consumo de AINEs se asocia a un caso de muerte cada 1.200 pacientes tratados con AINEs durante 2 meses (9) y que en los Estados Unidos el número de muertes por AINEs se considero similar a otras patologías como el SIDA, y superior al la originada por el asma o el cáncer de cervix, patologías a las que obviamente se emplean también importantes recursos económicos (10). En España se ha estimado que el número de muertes originados por el consumo de AINEs es superior a los 1200 casos anuales (8). En lo que se refiere a España y sin tener que inferir necesariamente una relación causal directa, el aumento del gasto farmacéutico en esta partida se ha asociado, según datos propios del área sanitaria III de Zaragoza, a una reducción del $50 \%$ en nuevos diagnósticos de úlcera péptica en los últimos 15 años. Igualmente se ha observado una reducción del $40 \%$ en la tasa de complicaciones a pesar de haber subido en un $28 \%$ el consumo de AINE (excluyendo los coxibs) y a pesar de haberse objetivado una reducción no mayor del 5,6\% en la frecuencia de infección del H. pylori en la población de entre 20-80 años.

Es también obvio que allá donde el sistema impone frenos al gasto por imperativos de presupuesto, que deben explicarse a la población, la labor de los profesionales y la de los farmacoeconomistas es encontrar las situaciones clínicas donde la relación coste-beneficio sea máxima. Desde hace ya tiempo se viene señalando que en términos de coste-beneficio en este área, la población diana es la población que presenta factores de riesgo y que engloban fundamentalmente a pacientes con historia previa de úlcera péptica, edad superior a los 60-65 años, ltoma concomitante de 2 AINEs, incluyendo la aspirina a dosis bajas, toma de dosis altas de AINEs, y toma concomitante de anticoagulantes (warfarina) o corticosteroides (> 10 $\mathrm{mg} /$ prednisona día) (4). Si bien es cierto que definir los propios límites de los factores de riesgo (p. ej. edad, dosis, número de factores de riesgo,...) no es fácil, parece también obvio que la simple historia clínica es suficiente para estimar si un paciente se encuentra en ese grupo y ejecutar la oportuna acción terapéutica. Es en este punto donde la incorporación de nuevos AINEs (coxibs) con un perfil de seguridad gastrointestinal mayor que los AINEs clásicos pueden tener un mayor impacto en la reducción de costes y en mejorar la calidad de vida de los pacientes con patología osteoarticular.

A día de hoy, los datos disponibles señalan que los coxibs no inducen úlceras endoscópicas y se asocian, aun administrados a dosis altas, a una reducción manifiesta de la frecuencia de complicaciones que alcanzan el $50-60 \%$ cuando se han comparado a AINEs clásicos en estudios apropiados y prolongados en el tiempo (11-13). En estudios de menor duración y diseñados para responder diferentes objetivos, los datos acumulados señalan que la frecuencia de complicaciones es o se acerca a la objetivada en poblaciones control que tomaban placebo $(14,15)$. Aun señalando que existen todavía situaciones clínicas en las que se precisa mayor información (p. ej. uso concomitante con aspirina a dosis bajas) (16) y que su perfil de seguridad a otros niveles (ej renal) no es mejor que el de los AINES clásicos, el beneficio sobre estos en el tracto gastrointestinal no ofrece a día de hoy duda alguna. A este respecto es llamativo comprobar como durante el año 2000, en el área III de Zaragoza, a pesar de haber registrado un aumento del $19 \%$ en la prescripción de AINE respecto al año anterior, la incidencia de complicaciones decreció, hecho que pudo deberse, al menos en parte, a que ese aumento fue debido en un $88,3 \%$ de los casos a la prescripción de coxibs.

El beneficio de los coxibs sobre los AINEs clásicos esta presente tanto en la población de alto como de bajo riesgo, pero desde la óptica coste-beneficio esta situación debe plantearse especialmente en la de alto riesgo y frente a la alternativa clásica de AINE junto a gastroprotector. Dado que los sistemas de salud y las cargas de los costes farmacéuticos entre el usuario y los sistemas sanitarios son tan diferentes, cada situación exige un estudio individualizado. Aunque no es objetivo de este editorial analizar este aspecto de forma extensa, se ha de señalar que hasta el momento actual algunos análisis efectuados en diferentes países señalan, en su mayoría, un beneficio de la alternativa coxib sobre la combinación AINE clásico y gastroprotector en la población con factores de riesgo (17-22). Es obvio que son muchos los factores a considerar y no solo en costes directos sino también otros como mortalidad, calidad de vida, efectos secundarios a otros niveles, costes indirectos relacionados con las horas de trabajo, polifarmacia, etc., lo cual dificulta enormemente los análisis y las estimaciones. Es de destacar, sin embargo, que la muerte asociada a efecto adversos por fármacos esta directamente relacionada con el número de fármacos y la frecuencia de uso de los mismos $(1,2)$. Bajo esta perspectiva solo es ya bienvenida cualquier innovación que reduzca la carga de fármacos de nuestros pacientes. Los estudios clínicos y los farmacoeconómicos debieran además guiarnos en la toma de decisiones apropiadas.

\section{AGRADECIMIENTOS}

A la Dra. Ma Pérez-Aósa y el Dr. Miguel Siles por su inestimable ayuda en la obtención de la información referente al área III de Zaragoza.

\section{A. LANAS}

Servicio de Aparato Digestivo. Hospital Clínico Lozano Blesa. Zaragoza

\section{Bibliografía}

1. Lazarou J, Pomeranz BH, Corey PN.Incidence of adverse drug reactions in hospitalized patients: a meta-analysis of prospective studies. JAMA 1998; 279 (15):1200-5.
2. http://www.jr2.ox.ac.uk/bandolier/index.html
3. Kempen GI, Ormel J, Brilman EI, Relyveld J. Adaptive responses among Dutch elderly: the impact of eight chronic medical conditions on 
health-related quality of life. Am J Public Health 1997 Jan;87(1):38-44

4. Lanas A, Pique JM, Ponce J; Asociación Española de Gastroenterología. Estrategia clínica para el paciente que precisa antiinflamatgorios no esteroides. papel de los inhibidores de la COX-2. Gastroenterol Hepatol 2001; 24(1): 22-36. Erratum in: Gastroenterol Hepatol 2001 24(3): 134.

5. Vargas E, Cabrera L, Morón A, García M, Soto J, Rejas J. Complicaciones gastro-intestinales severas potencialmente relacionadas con el consumo de antiinflamatorios no esteroideos: coste del tratamiento hospitalario para el Sistema Nacional de Salud de nuestro país. An Med Interna (Madrid) 2001; 18: 564-568.

6. Lanas A. Cost stratification of nonsteroidal antiinflammatory drugassociated gastrointestinal side effects. Med Clin (Barc) 2000; 114 (supl 3): 46-53.

7. Lanas A, Arroyo MT. Efectos Secundarios gastrointestinales asociados al tratamiento con antiinflamatorios no esteroideos ¡Qué caros! En Actualizaciones en Gastroenterología: Prevencion de la gastropatía por AINE. Prous Science. Barcelona 2000; 59-66

8. Lanas A. Clinical experience with COX-2 inhibitors. Rheumatology 2001 (in press).

9. Tramer MR, Moore A, Reynolds DJM, McQuay HJ. Quantitative estimation of rare adverse events which follows a biological progression: a new model applied to chronic NSAID use. Pain 2000; 85: 169-182.

10. Wolfe MM, Lichtenstein DR, Singh G.Gastrointestinal toxicity of nonsteroidal antiinflammatory drugs. N Engl J Med 1999; 17: 340(24): 1888-99 Laine L, Harper S, Simon T, Bath R, Johanson J, Schwartz H et al. A randomized trial comparing the effect of rofecoxib, a cylooxigenase 2-specific inhibitor, with that of ibuprofen on the gastroduodenal mucosa of patients with osteoarthritis. Gastroenterology 1999; 117: 776-783.

11. Laine L, Harper S, Simon T, Bath R, Johanson J, Schwartz H et al. A randomized trial comparing the effect of rofecoxib, a cylooxigenase 2specific inhibitor, with that of ibuprofen on the gastroduodenal mucosa of patients with osteoarthritis. Gastroenterology 1999; 117: 776-783.

12. Simon LS, Lanza FL, Lipsky PE, Hubbard RC, Talwalker S, Schwartz BD et al. Anti-inflammatory and Upper Gastrointestinal Effects of Celecoxib in Rheumatoid Arthritis. A Randomized Controlled Trial. JAMA 1999; 282: 1921-1928.
13. Bombardier C, Laine L, Reicin A, Shapiro D, Burgos-Vargas R, Davis D, et al. Comparison of Upper Gastrointestinal Toxicity of Rofecoxib and Naproxen in Patients with Rheumatoid Arthritis. N Engl J Med 2000; 343: 1520-8.

14. Langman MJ, Jensen DM, Watson DJ, Harper SE, Zhao P-L, Bolognese JA. Adverse upper gastrointestinal effects of rofecoxib compared with NSAIDs. JAMA 1999; 282: 1929-33.

15. Goldstein JL, Silverstein FE, Agrawal NM, Hubbard RC, Kaiser J, Clement JM et al. Reduced risk of upper gastrointestinal ulcer complications with celecoxib, a novel COX-2 inhibitor. Am J Gastroenterol 2000; 95: 1681-1690.

16. Silverstein FE, Faich G, Goldstein JL, Simon LS, Pincus T, Whelton A et al. Gastrointestinal toxicity with celecoxib vs nonsteroidal antiinflammatory drugs for osteoarthritis and rheumatoid arthritis: the CLASS study: A randomized controlled trial. Celecoxib Long-term Arthritis Safety Study. JAMA 2000; 284: 1247-1255.

17. Peloso PM, Scheiman JM The economic implications of cyclooxygenase-2-specific inhibitors. Am J Med 2001 Feb 19;110 Suppl 3A:50S-4S.

18. Chancellor JV, Hunsche E, de Cruz E, Sarasin FP.Economic evaluation of celecoxib, a new cyclo-oxygenase 2 specific inhibitor, in Switzerland. Pharmacoeconomics 2001; 19 Suppl 1: 59-75.

19. Zabinski RA, Burke TA, Johnson J, Lavoie F, Fitzsimon C, Tretiak R Chancellor JV. An economic model for determining the costs and consequences of using various treatment alternatives for the management of arthritris in Canada. Pharmacoeconomics 2001; 19 Suppl 1: 49-58.

20. Burke TA, Zabinski RA, Pettit D, Maniadakis N, Maurath CJ, Goldstein JL. A framework for evaluating the clinical consequences of initial therapy with NSAIDs, NSAIDs plus gastroprotective agents, or celecoxib in the treatment of arthritis. Pharmacoeconomics 2001; 19 Suppl 1: 33-47.

21. Pellissier JM, Starus WL, Watson DJ, Kong SX, Harper SE. Economic evaluation of rofecoxib versus nonselective nonsteroidal anti-inflammatory drugs for the treatment of osteoarthritis. Clin Ther 2001; 23: 106179.

22. Sundy JS.Cox-2 inhibitors in rheumatoid arthritis. Curr Rheumatol Rep 2001; 3(1): 86-91. 